\title{
Novel Dipole Trapped Spheromak Configuration
}

\author{
M. R. Brown, ${ }^{1, *}$ C. D. Cothran, ${ }^{1}$ J. Fung, ${ }^{1}$ M. J. Schaffer, ${ }^{2}$ and E. Belova ${ }^{3}$
}

\begin{abstract}
We report the observation and characterization of a spheromak formed in the Swarthmore Spheromak Experiment (SSX) and trapped in a simple dipole magnetic field. The spheromak is studied in the prolate (tilt unstable) $0.4 \mathrm{~m}$ diameter, $L=0.6 \mathrm{~m}$ length copper flux conserver in SSX. This configuration is stable to the tilt, despite the prolate flux conserver. The spheromak is characterized by a suite of magnetic probe arrays for magnetic structure $\mathbf{B}(r, t)$, ion Doppler spectroscopy for $T_{i}$ and flow, interferometry for $n_{e}$, and soft X-ray analysis for $T_{e}$. Three dimensional MHD simulations of this configuration verify its gross stability.
\end{abstract}

KEY WORDS: Magnetic confinement; spheromak; dipole.

\section{INTRODUCTION}

Spheromaks $[1,2]$ are high $\beta$, compact, translatable, simply connected plasma configurations all of which properties make them an attractive concept for a fusion reactor. When spheromaks form, poloidal flux is amplified during the relaxation process [3]. The spheromak is unstable to an ideal MHD tilt [4] which must be stabilized in order to be a viable fusion reactor. Spheromaks are typically stabilized by closefitting flux conservers [3,4] or by arrays of external coils [5]. In order for a cylindrical flux conserver to be stabilizing, it must be oblate with length-to-radius ratio $L / R \leq 1.67$ [6-8].

We have been studying novel configurations in a new prolate (tilt unstable) $0.4 \mathrm{~m}$ diameter, $0.6 \mathrm{~m}$ length, $3 \mathrm{~mm}$ wall copper flux conserver at the Swarthmore Spheromak Experiment. Initial results

${ }^{1}$ Department of Physics and Astronomy, Center for Magnetic Self Organization Swarthmore College, Swarthmore, PA, 19081, USA.

2 General Atomics85608, San Diego, CA, 92186, USA.

3 Plasma Physics Laboratory, Princeton University, Princeton, NJ, 08543, USA.

* To whom correspondence should be addressed. E-mail: doc@swarthmore.edu in this new flux conserver were presented in Cothran et al. [9]. Injection of a single spheromak into a vacuum dipole magnetic field generated with current in the same sense as the toroidal current in the spheromak generates an axisymmetric toroidal structure that is stable for the lifetime of the object, despite the elongated flux conserver.

Global magnetic structure of dipole-trapped spheromaks is studied with up to 600 individual internal magnetic probes operated simultaneously at 1.25 MHz using a multiplexer system [10]. We have used primarily distributed, coarse resolution arrays for these studies. Line averaged electron density is monitored with a quadrature $\mathrm{HeNe}$ laser interferometer [11]. For these studies, the single interferometer chord is placed on a diameter and at up to three axial locations (east, west, and midplane). We can scan density in a range $n_{e}=2-6 \times 10^{14} \mathrm{~cm}^{-3}$. Line averaged electron temperature $\left(T_{e} \cong 15 \mathrm{eV}\right)$ is inferred from a model fit to data from a soft X-ray array.

In addition, line averaged ion flow and temperature $T_{i}$ at the midplane is monitored with a $1.33 \mathrm{~m}$ ion Doppler spectrometer (IDS) for this configuration. We achieve high spectral resolution $(0.0075 \mathrm{~nm}$ per pixel) with an Echelle grating operating at 25th order [12]. We achieve high temporal resolution by 
using a 32 channel photomultiplier tube array. The SSX IDS instrument measures with $1 \mu$ s or better time resolution the width and Doppler shift of the $C_{I I I}$ impurity (H plasma) $229.7 \mathrm{~nm}$ line to determine the temperature and line-averaged flow velocity during spheromak trapping. The instrument design temperature is approximately $3 \mathrm{eV}$ for $C$ emission lines. Velocity resolution is about $5 \mathrm{~km} / \mathrm{s}$, corresponding to approximately $0.05 v_{A}$.

In Section "Dipole trapped spheromak", the detailed properties of the dipole-trapped spheromak are discussed in turn (magnetics and flow). A summary is presented in Section "Summary".

\section{DIPOLE TRAPPED SPHEROMAK}

Early attempts at stabilizing the tilt included the use of external coils in the Princeton S1 spheromak [13] and a close fitting copper flux conserver in the Los Alamos CTX spheromak [4]. These early experiments met with limited success unless an oblate flux conserver $(L / R \leq 1.67)$ was employed [2]. In modern spheromaks (for example, SSPX [5]), a closefitting, oblate $(L / R \cong 1)$ flux conserver is used in conjunction with arrays of shaping coils.

The SSX apparatus uses opposing, coaxial magnetized plasma guns at either end of a cylindrical copper flux conserver to produce spheromaks of either handedness (see Figure 1). Several different flux conservers have been used at SSX over the years with diameters varying between 0.17 and $0.5 \mathrm{~m}$. The present flux conserver diameter is $0.40 \mathrm{~m}$ with a length of $0.61 \mathrm{~m}$ (aspect ratio or elongation of 1.5 and $L / R=3$ ). The aspect ratio is deliberately chosen so that a spheromak should be tilt unstable $(L / R>1.67)$ for basic studies. Single spheromaks have previously been studied in SSX in oblate, tilt stable flux conservers [3]. The guns are typically operated at $1.0 \mathrm{mWb}$ of magnetic flux but during the plasma relaxation into a spheromak, poloidal flux is typically amplified to $\sim 3 \mathrm{mWb}$.

\section{Magnetics}

A pair of trapping coils at the midplane were used to produce the dipole field. The trapping coils have a mean diameter of $0.50 \mathrm{~m}$ and had a mean separation of $0.063 \mathrm{~m}$ (see Figure 1). A pair of coils was to provide a diagnostic gap at the midplane. Each coil was housed in its own welded vacuum jacket. The on-axis field of these coils can be varied from 0 to $750 \mathrm{G}$ in both directions. The sign of the field used for our dipole trapping studies was such that the trapping coil current and the spheromak toroidal current were in the same sense; attractive. Oppositely directed image currents in the flux conserver walls complete the equilibrium.

In Figure 2, we show the global magnetic structure of an approximately axisymmetric $m=0$ spheromak trapped in an externally applied dipole field at the midplane. The dipole field strength was about $150 \mathrm{G}$ field on axis (about $5 \mathrm{kA}$ turns of total current in the coils). Side and top views are depicted above, while the three axial views (called east, midplane, west) are depicted below. Each chord of

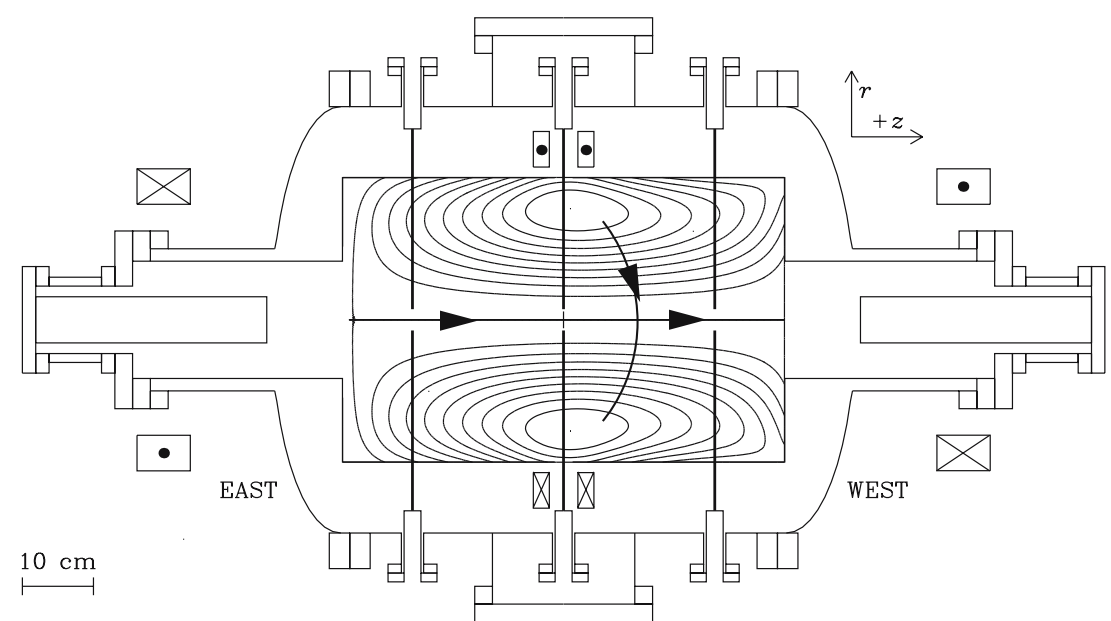

Fig. 1. Swarthmore spheromak experiment. The flux conserver is $0.4 \mathrm{~m}$ in diameter and $0.6 \mathrm{~m}$ in length. Schematic showing orientation of single prolate spheromak equilibrium calculated from a Grad-Shafranov solver and location of distributed magnetic probe array. Dipole trapping coil is at the midplane. 

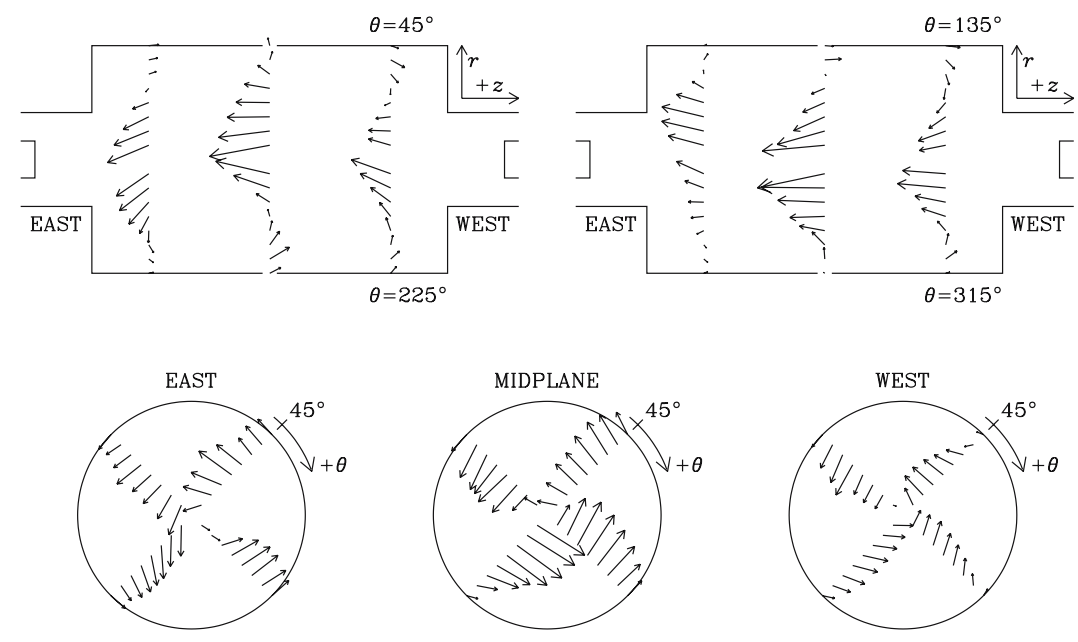

$1.0 \mathrm{kG}$

$62.4 \mu \mathrm{s}$

Fig. 2. Global magnetic structure of dipole trapped spheromak. Five views of the data are shown: two orthogonal $r-z$ projections (top row), $3 r-\theta$ projections (bottom row) at $62.4 \mu \mathrm{s}$. The scale of $1.0 \mathrm{kG}$ is indicated bottom left.

magnetic data is measured by a linear array of probes measuring $B_{r}, B_{z}, B_{\theta}$ at $25 \mathrm{~mm}$ resolution radially. Data for this shot were obtained at three axial locations, four azimuthal locations, and eight radii (96 simultaneous measurements of vector B). The time presented for this single spheromak shot is well into the relaxation and decay phase $(62.4 \mu \mathrm{s})$. A movie of the time history of this configuration shows little dynamics. Evidently, the combination of the attractive external dipole field, the close-fitting copper flux conserver, and perhaps line-tying stabilize the tilt. Note also that the trapped plasma encircles a large flux core. Some of this flux is line-tied to the copper end-plates of the flux conserver. Remarkably, this plasma does not tilt and remains in a nearly axisymmetric configuration for the duration of the discharge. Spheromaks formed in this flux conserver without the dipole field either by co-helicity merging or with a single gun tilt within a few $\mu s$.

Note that the Grad-Shafranov equilibrium configuration shown in the schematic with Figure 1 was calculated with characteristics matched to those measured in the experiment. The boundary conditions for the calculated equilibrium are the same as in the experiment: highly conducting cylindrical walls with $0.4 \mathrm{~m}$ diameter, $0.6 \mathrm{~m}$ length, and midplane trapping coils with a total of $5 \mathrm{kA}$ of current providing about $150 \mathrm{G}$ field on axis. We have chosen a broad current profile for the equilibrium $(50 \mathrm{kA}$ total toroidal plasma current) and $\beta \cong 0.2$. Note that the measured structure in Figure 2 closely resembles this calculated equilibrium. Both have $0.1 T$ field on the geometric axis with the magnetic axis at about $r=0.17 \mathrm{~m}$.

In Figure 3, we show a plot of the axial field at the midplane $B_{z}(r)$ for the same shot and time as Figure 2 compared with the theoretical prediction from the force-free theory [3]:

$$
B_{z}(r)=B_{0} J_{0}\left(k_{r} r\right) k_{r}=\frac{3.8317}{R}
$$

where $R=20 \mathrm{~cm}$ in our case. In addition, the $B_{z}(r)$ data from the Grad-Shafranov equilibrium in Figure 1 is also plotted (50 kA total toroidal plasma current and $\beta \cong 0.2$ ). The $B_{z}(r)$ data (square symbols)

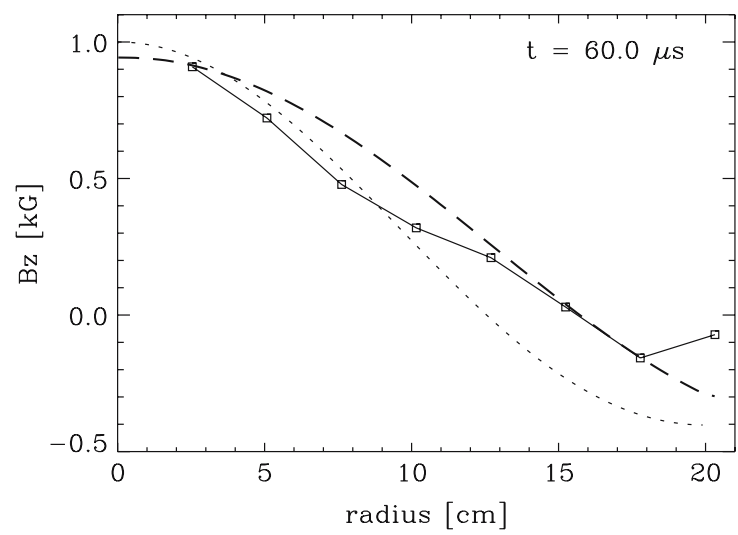

Fig. 3. Comparison of $B_{z}(r)$. Data from figure 2 (symbols) are compared with force-free Bessel model (dotted) and a Grad-Shafranov equilibrium model (dashed) with $\beta=0.2$ and $5 \mathrm{kA}$ in the dipole coils (from Figure 3). The $B_{z}(r)$ data are from $60.0 \mu \mathrm{s}$ and the four azimuthal arrays are averaged. 
are from $60.0 \mu$ s and the four azimuthal arrays are averaged ( $m=0$ symmetry). The experimentally measured magnetic axis ( $B_{z}$ reversal) for this configuration $(0.75 \mathrm{R})$ is significantly outboard of the force-free prediction $(0.62 \mathrm{R})$. This is not surprising since our dipole-trapped spheromak has significant thermal pressure $(\beta \cong 0.2)$. Note that the finite $\beta$ equilibrium is a better match to the data than the simple Bessel model at larger radii.

\section{Flow and heating}

Ion flow and temperature of the dipole trapped SSX spheromaks have been measured with an ion Doppler spectrometer at the midplane [12]. Lineshapes from $C_{I I I}$ emission were measured during the formation and decay of the dipole-trapped spheromak. The chords for these measurements are at the midplane as shown in Figure 1. Moment analysis of the lineshapes were performed. Shifts of the mean corresponding to radial flow speeds of $\leq 10 \mathrm{~km} / \mathrm{s}$ are observed in occasional bursts. However, the striking aspect of the flow data is the relatively low average flow speeds in this configuration (less than $10 \mathrm{~km} / \mathrm{s}$ throughout most of the plasma for most of the discharge). In contrast, radial and azimuthal flows up to $40 \mathrm{~km} / \mathrm{s}$ have been measured in SSX during spheromak merging experiments.

Ion temperature extracted from the second moment of the lineshape data show heating up to $T_{i} \cong 30 \mathrm{eV}$. In Figure 4, we plot the mean $C_{I I I}$ ion temperature from 10 shots across the full $0.4 \mathrm{~m}$ diameter at the midplane. Note evidence of ion heating during relaxation (35-45 $\mu \mathrm{s})$ followed by

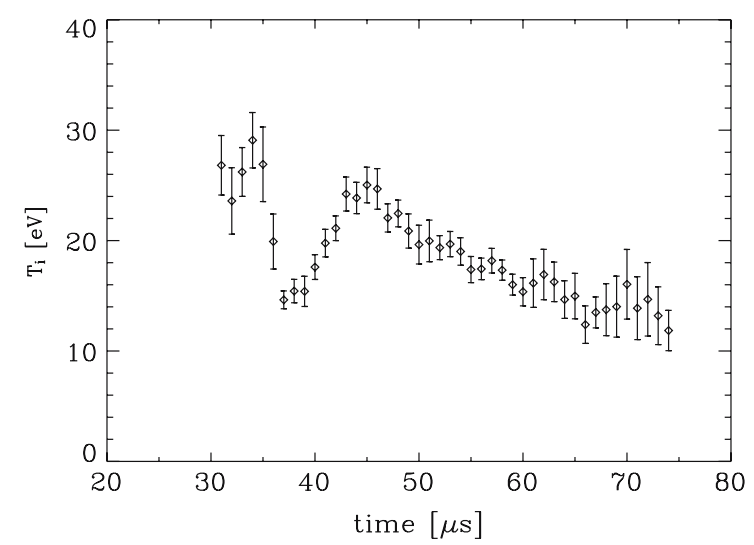

Fig. 4. Mean ion temperature (IDS view chord is along a diameter) from 10 shots at the midplane as a function of time for dipole trapped spheromak. Note evidence of ion heating during relaxation (35-45 $\mu \mathrm{s})$ followed by cooling during decay.

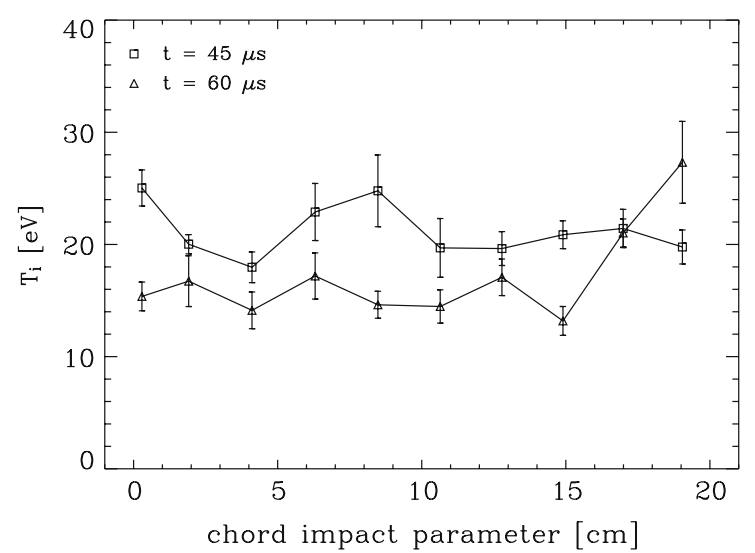

Fig. 5. Mean ion temperature as a function of impact parameter for dipole trapped spheromak at 45 and $60 \mu \mathrm{s}$.

cooling during decay. In Figure 5, we plot the mean $C_{I I I}$ ion temperature from 10 shots along the line of sight for each 10 chords, binned in $5 \mu$ s intervals to smooth shot-to-shot variability. Profiles at 45 and $60 \mu \mathrm{s}$ are shown. Note that this data contains information about dynamical velocity fluctuations as well as thermal temperature. These data are not inverted. There is evidence of heating from 10 up to $25 \mathrm{eV}$ during the relaxation phase (at around $45 \mu \mathrm{s}$ ) for most of the bulk of the plasma $(r=0-15 \mathrm{~cm})$. Note also that the temperature profiles are relatively flat.

\section{SUMMARY}

Stable, axisymmetric spheromaks have been formed and stably trapped in the combination of a simple, static dipole magnetic field and conducting wall image currents at SSX. The Grad-Shafranov equilibria are calculated to approximately fit the magnetic and pressure data and thus delineate the possible size and shape of the spheromak. The spheromak remains stable for the lifetime of the discharge despite the large elongation of the flux conserver $(L / R=3>1.67)$. Azimuthal flow velocities measured with ion Doppler spectroscopy are relatively low $(10 \mathrm{~km} / \mathrm{s})$ and ion temperature peaks at about $T_{i}=25 \mathrm{eV}$. The magnetic field on axis is about $0.1 \mathrm{~T}$, density about $2 \times 10^{14} \mathrm{~cm}^{-3}$, so $\beta \cong$ 0.25 . A fully three dimensional MHD simulation has verified the gross stability of the configuration.

\section{ACKNOWLEDGMENTS}

The authors gratefully acknowledge the assistance of V. Swisher and D. Cohen. 


\section{REFERENCES}

1. M. R. Brown, Phys. Plasmas, 6, 1717 (1999).

2. T. R. Jarboe, Plasma Phys. Contr. Fusion, 36, 945 (1994).

3. C. G. R. Geddes, T. W. Kornack, and M. R. Brown, Phys. Plasmas, 5, 1027 (1998).

4. T. R. Jarboe, I. Henins, H. W. Hoida, R. K. Linford, J. Marshall, D. A. Platts, and A. R. Sherwood, Phys. Rev. Lett., 45, 1264 (1980).

5. H. S. McLean, S. Woodruff, E. B. Hooper, et al., Phys. Rev. Lett., 88, 125004 (2002).

6. P. M. Bellan, Spheromaks (Imperial College Press, 2000).

7. A. Bondeson, G. Marklin, Z. G. An, H. H. Chen, Y. C. Lee, and C. S. Liu, Phys. Fluids, 24, 1682 (1981).
8. J. M. Finn, W. M. Manheimer, and E. Ott, Phys. Fluids, 24, 1336 (1981).

9. C. D. Cothran, A. Falk, A. Fefferman, M. Landreman, M. R. Brown, and M. J. Schaffer, Phys. Plasmas, 10, 1748 (2003).

10. M. Landreman, C. D. Cothran, M. R. Brown, M. Kostora, and T. Slough J., Rev. Sci. Instrum., 74, 2361 (2003).

11. M. R. Brown, C. D. Cothran, M. Landreman, D. Schlossberg, and W. H. Matthaeus, Astrophys. J. Lett., 577, L63 (2002).

12. C. D. Cothran, J. Fung, M. R. Brown and M. J. Schaffer, Fast, High Resolution Echelle Spectroscopy of a Laboratory Plasma (Rev. Sci. Instrum. submitted, 2006).

13. M. Yamada, H. P. Furth, W. Hsu, et al., Phys. Rev. Lett., 46, 188 (1981) 